\title{
Prospects for Chiral Nonlinear Optical Media
}

\author{
Victor Ostroverkhov, Oksana Ostroverkhova, Rolfe G. Petschek, Kenneth D. Singer, Ludmila Sukhomlinova, and \\ R. J. Twieg
}

Invited Paper

\begin{abstract}
This paper describes the development and optimization of chiral, nonpolar media with large second-order nonlinear optical responses. We employ molecular engineering, quantum-mechanical sum-over-states theory, and measurements of molecular hyperpolarizability by means of Kleinman-disallowed hyper-Rayleigh scattering in order to understand molecular properties. Then we analyze the appropriate arrangement of the chromophores that produce an optimum axial nonlinear optical medium. Chromophores with large Kleinman disallowed traceless symmetric second-rank tensor hyperpolarizabilities $\beta$ can be aligned so as to result in large susceptibilities $\chi^{(2)}$ in structures that lack polar order. We found that $\Lambda$-shaped chromophores with $C_{2 v}$ or similar symmetry are good candidates for these materials, as they can exhibit large second-rank components of the hyperpolarizability tensor. A wide variety of techniques can be used to fabricate bulk materials belonging to the chiral nonpolar symmetry groups $D_{\infty}$ and $D_{2}$. The microscopic chromophore alignment schemes that optimize the nonlinear optical response in such materials are deduced from general symmetry consideration for both molecules and bulk. We also speculate on the possible application of such materials as high-bandwidth spatial light modulators.
\end{abstract}

Index Terms-Electrooptic materials, liquid crystals, organic nonlinear optical materials, polymers.

\section{INTRODUCTION}

$\mathbf{S}$ ECOND-ORDER nonlinear optics requires a noncentrosymmetric material. Consequently, much effort has gone into the development of polar materials that exploit the dipolar (vector) component of the molecular hyperpolarizability based on a push-pull conjugated model [1], [2]. However, it has been recognized for a long time that even isotropic fluids containing chiral molecules can exhibit second-order nonlinear optical effects [3]. Second harmonic generation is not observed in such isotropic chiral media, but it may be observed in chiral materials that are at least uniaxially aligned. Since dipoles have low entropy when they are aligned and also, generally, higher energy when aligned parallel to each other, it is generally difficulty to align and maintain polar alignment in macroscopic materials. This consideration leads to compromises in nonlinear optical response due to constraints on chromophore density and

Manuscript received April 11, 2001. This work was supported by the National Science Foundation under the Advanced Liquid Crystalline Optical Materials (ALCOM) Science and Technology Center DMR89-20147.

V. Ostroverkhov, O. Ostroverkhova, R. G. Petschek, and K. D. Singer are with Case Western Reserve University, Cleveland, OH 44106 USA.

L. Sukhomlinova and R. J. Twieg are with Kent State University, Kent, OH 44242 USA.

Publisher Item Identifier S 1077-260X(01)11224-4. alignment, as well as difficulties in producing stable high-optical and mechanical-quality materials. In contrast, nonpolar axial alignment results in many materials from simple steric interactions, or by stretching of polymers. Many such media lacking full rotation symmetry and mirror planes will have allowed components of the first hyperpolarizability. Chiral media where mirror symmetries are absent, together with uniaxial $\left(D_{\infty}\right)$ or biaxial $\left(D_{2}\right)$ alignment, are consistent with second-order nonlinear polarizabilities, including ones such as second harmonic generation and the linear electrooptical effect. Such materials can be easily fabricated by aligning chromophores in media that are expected to have relatively little light scattering. Because of this and intrinsic interest in chirality in general, there has been a recent increase of activity involving the macroscopic nonlinear optical properties of chiral materials [4]-[9]. However, work that has appeared discussing the molecular aspects necessary for obtaining a large nonlinear response in chiral media has mainly focused on polar media [10], [11].

We have recently described a scheme to optimize molecular properties of multidimensional nonlinear optical (NLO) chromophores for use in chiral nonlinear materials using molecular engineering, and including sum-over-states quantum-mechanical calculations and measurements of the rotational invariants of molecular hyperpolarizability $\beta$ by means of Kleinman-disallowed hyper-Rayleigh scattering [12]. Alternative nonpolar methods of alignment exploit the tensorial nature of the molecular hyperpolarizability $\beta$. Generally, the second harmonic hyperpolarizability tensor can be decomposed into four components, two of which transform under three-dimensional rotations as traceless symmetric secondand third-rank tensors. These components of higher rank are responsible for macroscopic second-order nonlinear properties of materials with nonpolar order. Optimization of the third-rank (or octupolar) hyperpolarizability has been addressed by others (e.g., [13]), while utilization of the second-rank component, which exists only in species with Kleinman symmetry (full index permutation) breaking, is addressed in this paper.

To have Kleinman-disallowed hyperpolarizabilities, a chromophore must have electrons that move in at least two dimensions. The figures of merit corresponding to the various irreducible components can be measured by means of Kleinmandisallowed hyper-Rayleigh scattering (KD-HRS) [14], [15]. We have examined a promising class of molecules, $\Lambda$-shaped $\pi$-conjugated chromophores that consist of two electron donors (or 
acceptors) at the ends connected to one acceptor (or donor) at the $\Lambda$ vertex. Crystal Violet, which may have a symmetry similar to $D_{3}$ is also interesting. Both have shown strong nonvector components of the hyperpolarizability tensor.

Once the chromophore is optimized for the second-rank tensor component, the next step is to properly embed it into a chiral axial medium. The symmetries $D_{\infty}$ and $D_{2}$ are important cases for nonpolar axial alignment, as we believe that these are easy to fabricate and occur naturally in liquid crystals, for example. Given a chromophore that has a Kleinman-disallowed irreducible hyperpolarizability component, the criteria for axial alignment that efficiently utilize the molecular nonlinearity can be enumerated. We note that chirality of the bulk does not necessarily require chirality of the chromophores, so that we will discuss how achiral (but Kleinman-asymmetric) molecules can be arranged in a chiral fashion that will result in an efficient NLO medium.

In the following sections of this paper, we discuss in detail the optimization of chiral NLO media and present recent results of Kleinman-disallowed HRS for a number of $\Lambda$-shaped molecules as chromophores for chiral nonpolar NLO media. We also speculate that such materials, when fully optimized, may be applicable to high-bandwidth spatial light modulators that exploit the fast electronic nonlinear optical response.

\section{CHROMOPHORE CHARACTERIZATION: KLEINMAN DisAllowed CASE}

\section{A. Irreducible Approach}

To begin to understand how to optimize the molecular properties that can be exploited in chiral media, we express the molecular hyperpolarizability in terms of the irreducible representation of the rotation group. The hyperpolarizability is a rank-3 tensor that transforms as the product of three rank-1 vectors. This can be reduced through the formalism of the addition of angular momenta [16]. In the most general case of parametric light scattering, the hyperpolarizability $\beta_{\mathrm{pls}}$ transforms as follows:

$$
\begin{aligned}
\beta_{\mathrm{pls}} & \sim 1 \otimes 1 \otimes 1 \\
& \sim 0 \oplus(1 \oplus 1 \oplus 1) \oplus(2 \oplus 2) \oplus 3 .
\end{aligned}
$$

Equation (1) shows that this $\beta$ consists of one antisymmetric pseudoscalar $(L=0)$, one fully symmetric rank-3 tensor $(L=$ $3)$, three vectors $(L=1)$, and two pseudotensors $(L=2)$. One of the rank-1 objects is fully symmetric; the remaining two rank-1 and rank-2 components transform as the mixed representation of the permutation group of three objects. The second harmonic hyperpolarizability is a third-rank tensor symmetric under permutation of the last two indexes, leading to a simpler analog to (1)

$$
\begin{aligned}
\beta_{\mathrm{SHG}} & \sim 1 \otimes(1 \otimes 1)_{\mathrm{sym}} \\
& \sim 1 \otimes(0 \oplus 2) \\
& \sim 1 \oplus 1 \oplus 2 \oplus 3 .
\end{aligned}
$$

In both (1) and (2), the hyperpolarizability tensor is presented formally as a direct sum of the irreducible parts whose components do not mix under three-dimensional rotation. Group theory provides a mechanism for extracting irreducible parts of $\beta$ in Cartesian form based on the fact that they exhibit different index permutation symmetry. In Cartesian coordinates, the decomposition of $\beta_{\mathrm{SHG}}(2)$ can be written as follows:

$$
\beta_{i j k}=\beta_{i j k}^{(3 s)}+\beta_{i j k}^{(2 m)}+\beta_{i j k}^{(1 s)}+\beta_{i j k}^{(1 m)} .
$$

Two components ( $1 \mathrm{~s}$ and $1 \mathrm{~m}$ ) transform as vectors, $2 \mathrm{~m}$ as a second-rank traceless symmetric pseudotensor, and $3 s$ as a third-rank traceless symmetric tensor. The indexes $s$ and $m$ denote the parts that are fully symmetric $(s)$ and those that have mixed $(m)$ symmetry under permutations. While there are a number of approaches to extracting rotationally irreducible components, in Cartesian representation this can be done by contracting the tensor $\beta$ with the fully symmetric Kroneker $\delta$ tensor and the fully antisymmetric Levi-Civita tensor $\varepsilon$. The components of lower rank are appropriately embedded in rank-3 tensor form.

In the fully symmetric case of Kleinman (full permutation) symmetry [17], the hyperpolarizability consists of just two components

$$
\beta_{\text {sym }} \sim 1 \oplus 3
$$

reflecting the two components that are fully symmetric under permutation.

Each irreducible component of the hyperpolarizability tensor in the decomposition given in (3) has a scalar invariant associated with it, which can serve as a natural measure of the component's contribution to the total $\beta$. Since there are two components of the same rank in the decomposition [(3)] (1s and $1 \mathrm{~m}$ ), there is also a complex-valued invariant defined as a dot product between the two vectors so that there are six (real) scalar rotational invariants that can be extracted from the hyperpolarizability tensor. These invariants can be measured in a specially designed hyper-Rayleigh scattering experiment, making it a powerful tool for characterizing chromophores for chiral NLO, as we describe later.

\section{B. Quantum-Mechanical Sum Analysis}

The method for optimizing molecular hyperpolarizabilities and the $L=2$ component of interest here can be understood by considering the quantum mechanical expressions for the tensors that transform like various irreducible representations of the rotation group, that is, like various traceless symmetric tensors. The expression for $\beta$ in terms of microscopic parameters of the molecule can be written down as a perturbative solution to the appropriate Schrödinger equation [18]

$$
\begin{array}{r}
\beta_{i j k}(2 \omega ; \omega, \omega) \\
=\frac{1}{2 \hbar^{2}} \sum_{m, n}\left(\frac{\mu_{g n}^{i}\left(\mu_{n m}^{j} \mu_{m g}^{k}+\mu_{n m}^{k} \mu_{m g}^{j}\right)}{\left(\omega_{n g}-2 \omega\right)\left(\omega_{m g}-\omega\right)}\right. \\
+\frac{\mu_{n m}^{i}\left(\mu_{g n}^{j} \mu_{m g}^{k}+\mu_{g n}^{k} \mu_{m g}^{j}\right)}{\left(\omega_{n g}^{*}+\omega\right)\left(\omega_{m g}-\omega\right)} \\
\left.+\frac{\mu_{m g}^{i}\left(\mu_{g n}^{j} \mu_{n m}^{k}+\mu_{g n}^{k} \mu_{n m}^{j}\right)}{\left(\omega_{n g}^{*}+\omega\right)\left(\omega_{m g}^{*}+2 \omega\right)}\right) .
\end{array}
$$


Equation (5) is a simplified form for the special case of second harmonic generation as reflected by the intrinsic symmetry with respect to permutation of the last two indexes. The ground state is denoted by $g$, while $n$ and $m$ run over all available quantum states. Dissipation is taken into account by introducing complex-valued transition frequencies $\omega_{n g} \equiv \omega_{n g}^{0}-i \Gamma_{n} / 2$, with $\omega_{n g}^{0}$ being a real transition frequency and $\Gamma_{n}$, the dissipation, being inversely proportional to the relaxation time. We can specialize this to the various representations of the rotation group, yielding (6), as shown at the bottom of the page, where $\Delta \vec{\mu}_{n g}=$ $\vec{\mu}_{n n}-\vec{\mu}_{g g}$ is the change in the dipole moment and $\mathrm{P}$ is the permutation operator that interchanges the indexes $i, j$, and $k$.

$$
\begin{aligned}
& \beta_{1 s}^{i}=\frac{1}{\hbar^{2}}\left\{\sum_{n \neq g} \frac{\omega_{n g}^{2}}{\left(\omega_{n g}^{2}-\omega^{2}\right)\left(\omega_{n g}^{2}-4 \omega^{2}\right)}\left(\Delta \mu_{n g}^{i}\left|\vec{\mu}_{g n}\right|^{2}+2 \mu_{g n}^{i}\left(\Delta \vec{\mu}_{n g} \bullet \vec{\mu}_{g n}\right)\right)\right. \\
& -\sum_{m \neq n \neq g} \sum_{n \neq g}\left[\frac{2 \omega^{4}\left(\omega_{m g}^{2}-4 \omega_{m g} \omega_{n g}+\omega_{n g}^{2}\right)+\omega^{2} \omega_{m g} \omega_{n g}\left(3 \omega_{m g}^{2}-\omega_{m g} \omega_{n g}+3 \omega_{n g}^{2}\right)-\omega_{m g}^{3} \omega_{n g}^{3}}{\left(\omega_{n g}^{2}-\omega^{2}\right)\left(\omega_{n g}^{2}-4 \omega^{2}\right)\left(\omega_{m g}^{2}-\omega^{2}\right)\left(\omega_{m g}^{2}-4 \omega^{2}\right)}\right. \\
& \left.\left.\times\left(\mu_{n m}^{i}\left(\vec{\mu}_{g n} \bullet \vec{\mu}_{g m}\right)+2 \mu_{g n}^{i}\left(\vec{\mu}_{n m} \bullet \vec{\mu}_{g m}\right)\right)\right]\right\} \\
& \beta_{1 m}^{i}=\frac{2 \omega^{2}}{\hbar^{2}}\left\{\sum_{n \neq g} \frac{2}{\left(\omega_{n g}^{2}-\omega^{2}\right)\left(\omega_{n g}^{2}-4 \omega^{2}\right)}\left[\vec{\mu}_{g n} \times\left[\vec{\mu}_{g n} \times \Delta \vec{\mu}_{n g}\right]\right]^{i}\right. \\
& +\sum_{m \neq n \neq g} \sum_{n \neq g} \frac{8 \omega^{4}-2 \omega^{2}\left(3 \omega_{m g}^{2}+2 \omega_{m g} \omega_{n g}\right)-\omega_{m g} \omega_{n g}\left(\omega_{m g}^{2}-\omega_{m g} \omega_{n g}-2 \omega_{n g}^{2}\right)}{\left(\omega_{n g}^{2}-\omega^{2}\right)\left(\omega_{n g}^{2}-4 \omega^{2}\right)\left(\omega_{m g}^{2}-\omega^{2}\right)\left(\omega_{m g}^{2}-4 \omega^{2}\right)} \\
& \left.\cdot\left[\vec{\mu}_{g n} \times\left[\vec{\mu}_{g m} \times \vec{\mu}_{n m}\right]\right]^{i}\right\} \\
& \beta_{2 m}^{\rho k}=\frac{3 \omega^{2}}{4 \hbar^{2}}\left\{\sum_{n \neq g} \frac{4}{\left(\omega_{n g}^{2}-\omega^{2}\right)\left(\omega_{n g}^{2}-4 \omega^{2}\right)}\left[\vec{\mu}_{g n} \times \Delta \vec{\mu}_{n g}\right]^{\rho} \mu_{g n}^{k}\right. \\
& +\sum_{m \neq n \neq g} \sum_{n \neq g}\left[\frac{2\left(2 \omega^{2}-\omega_{m g} \omega_{n g}-\omega_{n g}^{2}\right)}{\left(\omega_{n g}^{2}-\omega^{2}\right)\left(\omega_{n g}^{2}-4 \omega^{2}\right)\left(\omega_{m g}^{2}-\omega^{2}\right)}\left[\vec{\mu}_{n m} \times \vec{\mu}_{g n}\right]^{\rho} \mu_{g m}^{k}\right. \\
& \left.\left.-\frac{\left(2 \omega^{2}+\omega_{m g} \omega_{n g}\right)\left(\omega_{m g}^{2}-\omega_{n g}^{2}\right)}{2\left(\omega_{n g}^{2}-\omega^{2}\right)\left(\omega_{n g}^{2}-4 \omega^{2}\right)\left(\omega_{m g}^{2}-\omega^{2}\right)\left(\omega_{m g}^{2}-4 \omega^{2}\right)}\left[\vec{\mu}_{g m} \times \vec{\mu}_{g n}\right]^{\rho} \mu_{n m}^{k}\right]+\rho \leftrightarrow k\right\} \\
& \beta_{3 s}^{i j k}=\frac{1}{\hbar^{2}}\left\{\sum _ { n \neq g } \frac { \omega _ { n g } ^ { 2 } } { ( \omega _ { n g } ^ { 2 } - \omega ^ { 2 } ) ( \omega _ { n g } ^ { 2 } - 4 \omega ^ { 2 } ) } \left[\frac{1}{2} \mathrm{P}\left(\Delta \mu_{n g}^{i} \mu_{g n}^{j} \mu_{g n}^{k}\right)\right.\right. \\
& \left.-15\left(\left|\vec{\mu}_{g n}\right|^{2}\left(\Delta \mu_{n g}^{i} \delta^{j k}+\Delta \mu_{n g}^{j} \delta^{i k}+\Delta \mu_{n g}^{k} \delta^{i j}\right)+2\left(\Delta \vec{\mu}_{n g} \bullet \vec{\mu}_{g n}\right)\left(\mu_{g n}^{i} \delta^{j k}+\mu_{g n}^{j} \delta^{i k}+\mu_{g n}^{k} \delta^{i j}\right)\right)\right] \\
& -\sum_{m \neq n \neq g} \sum_{n \neq g} \frac{2 \omega^{4}\left(\omega_{m g}^{2}-4 \omega_{m g} \omega_{n g}+\omega_{n g}^{2}\right)+\omega^{2} \omega_{m g} \omega_{n g}\left(3 \omega_{m g}^{2}-\omega_{m g} \omega_{n g}+3 \omega_{n g}^{2}\right)-\omega_{m g}^{3} \omega_{n g}^{3}}{\left(\omega_{n g}^{2}-\omega^{2}\right)\left(\omega_{n g}^{2}-4 \omega^{2}\right)\left(\omega_{m g}^{2}-\omega^{2}\right)\left(\omega_{m g}^{2}-4 \omega^{2}\right)} \\
& \text { - }\left(\frac { 1 } { 2 } \mathrm { P } \left(\mu_{g n}^{i} \mu_{n m}^{j} \mu_{g m}^{k}-\frac{1}{5}\left[\left(\vec{\mu}_{g n} \bullet \vec{\mu}_{g m}\right)\left(\mu_{n m}^{i} \delta^{j k}+\mu_{n m}^{j} \delta^{i k}+\mu_{n m}^{k} \delta^{i j}\right)\right.\right.\right. \\
& \left.\left.\left.+2\left(\vec{\mu}_{n m} \bullet \vec{\mu}_{g m}\right)\left(\mu_{g n}^{i} \delta^{j k}+\mu_{g n}^{j} \delta^{i k}+\mu_{g n}^{k} \delta^{i j}\right)\right]\right)\right\}
\end{aligned}
$$



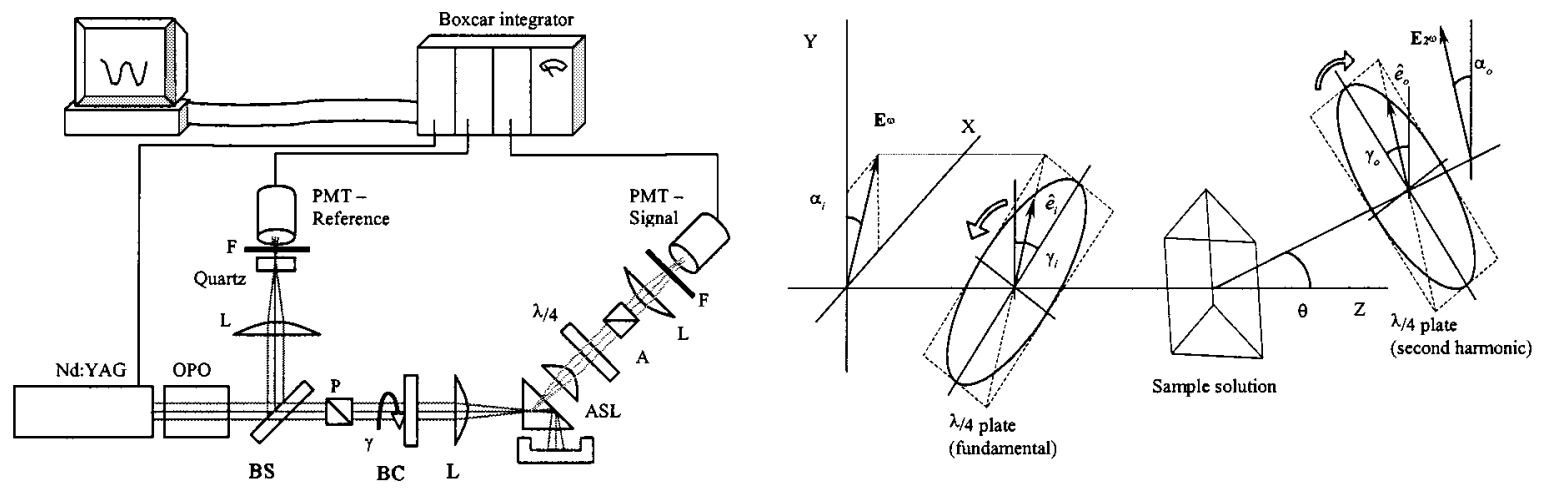

Fig. 1. Kleinman-disallowed hyper-Rayleigh scattering. (a) Experimental setup (P: input polarizer;, BS: Beamsplitter; L: Lens; BC: Berek compensator; ASL: Aspherical lens; $\lambda$ /4: Quarter-wave plate, A: Output polarizer; PMT: Photomultiplier tube; F: Filter). (b) Experiment geometry with arbitrary ellipticity of incident and detected light.

The first term in each case contains the two-level contributions, which are well known to give good descriptions of the nonlinear optical response in many quasi-one-dimensional charge-transfer molecules [19]. In general, a multilevel model must be applied, and so this general case will be considered first. Of particular interest is the appearance of both dot and cross products in the various terms. For the $1 s$ components, the dot product appears throughout, and thus the largest components are obtained if the various moments for important states are parallel. This feature is well known in optimizing quasi-one-dimensional push-pull molecules for polar nonlinear optics. The $3 s$ component involves differences including various dot products. Moreover, there are advantages to having more than one polarization of light relative to the molecule active in the nonlinear optical process. Moreover, there are sum rules involving the transition matrix elements in each direction so that $\beta$ must have components in more than one direction, with device structures in which this can be exploited. Thus, both multidimensional and linear molecules can have large and useful $3 s$ hyperpolarizabilities. We see, however, that cross products appear in the two Kleinman disallowed contributions, the $1 m$ and $2 m$ components, which implies that multidimensional molecules are a requirement. It is interesting to note that the $1 \mathrm{~m}$ component contains the cross product in the two-level terms and the dot product in the others, so that orthogonal moments favor the two-level terms while parallel moments favor the others. For the $2 m$ component of primary interest here, we see that orthogonal moments are always favored. Thus, the multidimensional behavior that optimizes this response involves states whose moments are orthogonal. Considering a single electronic excited state contributing in the Kleinman-disallowed part of $\beta$, one can write a two-state second-rank tensor as follows:

$$
\begin{aligned}
\beta_{(2 m)}^{i j}= & \frac{3 \omega^{2}}{\hbar^{2}\left(\omega_{n g}^{2}-\omega^{2}\right)\left(\omega_{n g}^{2}-4 \omega^{2}\right)} \\
& \cdot\left\{\left[\vec{\mu}_{n g} \times \Delta \vec{\mu}_{n g}\right]^{i} \mu_{n g}^{j}+\left[\vec{\mu}_{n g} \times \Delta \vec{\mu}_{n g}\right]^{j} \mu_{n g}^{i}\right\} .
\end{aligned}
$$

Even though the standard two-level model is not adequate for a description of the molecular response of two- and three-dimensional molecules, our analysis suggests that the hyperpolarizability components of interest to us in $\Lambda$-shaped (or quasi- $\Lambda$-shaped) molecules consisting of two donors and an acceptor (or vice versa) can be described by (7) in many cases, provided that the states with the correct symmetries are included in the sum [12]. For understanding the low-lying electronic states, $\Lambda$-shaped molecules that have a single conjugated region consisting of an apex and two identical "feet" can often be treated as having $C_{2 v}$ or $C_{2}$ symmetry. The electronic states of these molecules can be either symmetric ( $A$-type) or antisymmetric ( $B$-type) functions with respect to the $180^{\circ}$ rotation. This results in two distinct possibilities for the transition dipole moment: it must be either parallel (for an $A$ state) or perpendicular (for a $B$ state) to the molecular rotation axis, which corresponds to its dipole. Thus, according to (7), only the $B$-states will contribute to $\beta_{i j k}^{(2)}$ and, in fact, have the optimal geometry, since the $B$-state transition moment is orthogonal to the symmetry axis [12], [20]. To maximize the nonlinear optical response in chiral media composed of any $\Lambda$-shaped molecules, the lowest lying state should have substantial $B$-character. Semiempirical calculations for several $\Lambda$-shaped molecules indicate that some molecules of this type indeed do have quite strongly absorbing low-lying states with the appropriate symmetry [21]. In addition, the fact that $\beta^{(2 m)}$ and $\beta^{(1 m)}$ in (7) are proportional to $\omega^{2}$ indicates that the molecular response will be enhanced in the anomalous dispersion regime, e.g., for second harmonic generation when the fundamental laser frequency is lower energy, but the second harmonic is higher in energy than the energy of the relevant ( $B$-type) quantum state. In contradistinction, the (two level) contributions of a single quantum state (whether $A$ or $B$ type) to the Kleinman allowed vector or octupolar hyperpolarizabilities decreases in the anomalous dispersion regime.

\section{Kleinman-Disallowed Hyper-Rayleigh Scattering}

The experimental setup of a KD-HRS is shown in Fig. 1 and has been described in detail elsewhere [14], [15]. The light from an optical parametric oscillator tunable through the near infrared is sent through a polarizer and a quarter-wave plate, creating an arbitrary elliptical polarization, which is focused into a triangular quartz cell containing the chromophore in acetone solution. The scattered second harmonic light is collected at a $45^{\circ}$ angle and focused on a photomultiplier tube after a single elliptical polarization from the entire signal is selected with a sim- 

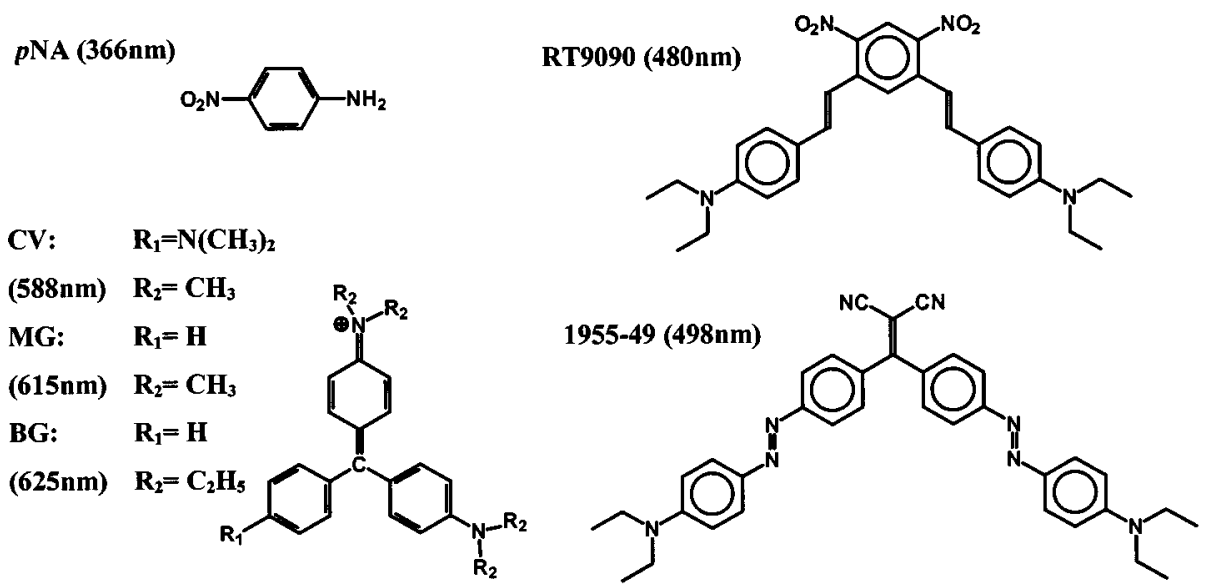

Fig. 2. Molecular structures of studied materials (with $\lambda_{\max }$ for each material in acetone solution).

ilar system of a quarter-wave plate and polarizer. In the experiment, the intensity of a definite outgoing second harmonic (elliptical) is recorded as a function of the polarization of the fundamental frequency light. The essential features of this experiment that distinguish it from a "traditional" HRS measurement of a Kleinman-symmetric hyperpolarizability tensor are 1) the scattering angle, which must not be $0^{\circ}, 90^{\circ}$, or $180^{\circ}$, and 2) the necessity to use elliptically polarized light in both the incident and the scattered beams. The values of the rotational invariants of $\beta$ are extracted from the signal by means of least square fitting [15]. The advantage of the analytic least square method used here is that the set of parameters (rotational invariants) that minimizes $\chi^{2}$ is determined analytically without the uncertainty associated with numerical gradient search methods.

Since most of our measurement wavelengths lie in the vicinity of molecular resonances, two-photon fluorescence may be a competing process that can complicate the measurements of $\beta$. To assure the dominance of the second harmonic signal over two-photon fluorescence, we measured the spectral content of the scattered light for all the chromophores at the laser excitation wavelengths used in this study. After verifying that two-photon fluorescence will not compete with the KD-HRS signal, we used narrow-band filters to block fluorescence at other wavelengths.

\section{Chromophores for Chiral Nonlinear Optics}

The molecular structures of the materials studied are shown in Fig. 2 [22]. Crystal Violet (CV), Malachite Green (MG), and Brilliant Green (BG) are well-known triarylmethane dyes. Crystal Violet is often considered to have a naïve three-fold symmetry. However, our measurements showed a rather strong contribution of vector components that are not allowed in nonpolar point groups such as $D_{3}$ or $D_{3 h}$, and this evidence suggests deviation from these symmetries in the ground state in these solvents. These results confirm other studies that suggest that CV is less symmetric than expected [23], [24]. Unlike Crystal Violet, Malachite Green and Brilliant Green have only two out of three phenyl rings substituted with amine donors, so that the resulting symmetry would be expected to be $C_{2 v}$ or, given the steric repulsions, $C_{2}$. Experimental results indicating deviation of Brilliant Green from Kleinman symmetry have been previously reported [25]. The two molecules synthesized in part for this study (Compounds RT9090 and 1955-49) are $\Lambda$-shaped chromophores with the acceptor in the middle and donors on the legs of $\Lambda$. These molecules are conventional linear and dipolar NLO chromophores except that they share a common central acceptor group that results in a $\Lambda$ geometry of the overall molecule. Table I summarizes the results of our measurements at the excitation wavelengths of 1560, 1340, 1064 , and $780 \mathrm{~nm}$. The figures of merit of the two-vector (1 ss and $1 \mathrm{~mm})$, the second-rank $(2 \mathrm{~mm})$, and the third-rank (3ss) components are defined as square roots of their scalar rotational invariants (see [15] for precise definitions). Absolute values of invariants were found through an external referencing scheme. Para-nitroaniline ( $p$ NA) was used as a reference substance at all studied wavelengths. The value of the Kleinman-allowed vector component of $p$ NA was derived from an EFISHG measurement at the longest wavelength $\left(\beta_{E F I S H}^{p N A}=12 \cdot 10^{-30}\right.$ esu at $1580 \mathrm{~nm}$ taken from [26, Ref. 114]) and scaled for the other wavelengths accordingly to two-level dispersion model [27]. One notices that all $\Lambda$-shaped molecules studied here possess sizable second-rank tensor components $\left(\beta_{2 \mathrm{~mm}}\right)$. Even in the relatively off-resonance, ordinary dispersion regime at 1560 -nm excitation, the values are comparable to the Kleinman symmetric ones.

\section{Chiral NonPolar Alignment fOR SeCond-Order NLO MATERIALS}

The decomposition into irreducible parts facilitates the analysis of $\chi^{(2)}$, as the latter is defined through rotational averages of $\beta$ [28]. The tensor parts in (3) belong to different irreducible representation of the permutation group of three objects and thus have different permutation symmetry. After being rotationally averaged, they will comprise the corresponding components of the macroscopic tensor $\chi$ with the same permutation symmetry that can be classified in a similar fashion

$$
\begin{aligned}
\chi_{I J K} \propto\left\langle\beta_{I J K}\right\rangle= & \left\langle\beta_{I J K}^{(3 s)}\right\rangle+\left\langle\beta_{I J K}^{(2 m)}\right\rangle+\left\langle\beta_{I J K}^{(1 m)}\right\rangle+\left\langle\beta_{I J K}^{(1 s)}\right\rangle \\
& \propto \chi_{I J K}^{(3 s)}+\chi_{I J K}^{(2 m)}+\chi_{I J K}^{(1 m)}+\chi_{I J K}^{(1 s)} .
\end{aligned}
$$

Thus, each irreducible component of bulk nonlinear susceptibility $\chi$ results from one component of the hyperpolarizability 
TABLE I

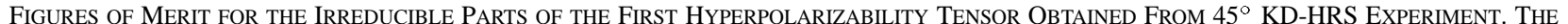

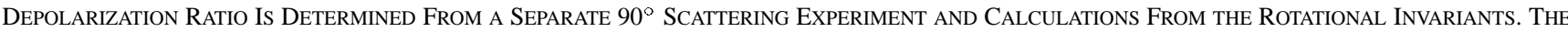

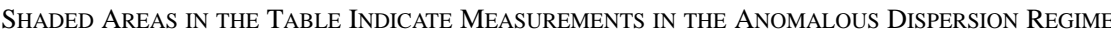

\begin{tabular}{|c|c|c|c|c|c|c|c|c|c|c|c|c|c|}
\hline \multirow[t]{2}{*}{ Material } & & \multicolumn{2}{|l|}{$\left\|\beta_{1 s s}\right\|$} & \multicolumn{2}{|l|}{$\left\|\beta_{1 \mathrm{~mm}}\right\|$} & \multicolumn{2}{|l|}{$\left\|\beta_{2 \mathrm{~mm}}\right\|$} & \multicolumn{2}{|l|}{$\left|\beta_{3 s s}\right|$} & \multicolumn{4}{|c|}{ Depolarization Ratio } \\
\hline & & & & & su $x 1$ & $\left.{ }^{-30}\right)$ & & & & $90^{\circ}$ Exper & nent & Calc. from & \\
\hline pNA (ref) & \multirow{4}{*}{\begin{tabular}{l}
$E$ \\
\multicolumn{5}{c}{} \\
$\stackrel{\circ}{\circ}$ \\
$\stackrel{2}{2}$
\end{tabular}} & 7.12 & \pm 0.16 & 0.0 & \pm 2.1 & 3.0 & \pm 2.6 & 4.95 & \pm 0.23 & 0.2 & \pm 0.01 & 0.24 & \pm 0.05 \\
\hline $\mathrm{CV}$ & & 83.5 & \pm 2.1 & 72.2 & \pm 2.1 & 84.1 & \pm 6.5 & 76.0 & \pm 4.2 & 0.63 & \pm 0.01 & 0.65 & \pm 0.06 \\
\hline MG & & 69.8 & \pm 1.8 & 14 & \pm 30 & 38.6 & \pm 9.5 & 54.0 & \pm 5.0 & 0.28 & \pm 0.02 & 0.28 & \pm 0.10 \\
\hline$B G$ & & 92.7 & \pm 2.1 & 24 & \pm 24 & 57.6 & \pm 6.1 & 68.0 & \pm 3.2 & 0.31 & \pm 0.02 & 0.30 & \pm 0.04 \\
\hline pNA (ref) & \multirow{3}{*}{ 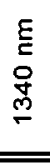 } & 8.09 & \pm 0.09 & 0.0 & \pm 2.4 & 3.6 & \pm 1.1 & 5.40 & \pm 0.19 & 0.22 & \pm 0.01 & 0.23 & \pm 0.06 \\
\hline RT9090 & & 266.8 & \pm 5.5 & 15 & \pm 41 & 128.8 & \pm 4.9 & 201.4 & \pm 4.6 & 0.27 & \pm 0.01 & 0.26 & \pm 0.01 \\
\hline $1955-49$ & & 316.0 & \pm 9.4 & 0 & \pm 98 & 180 & \pm 35 & 215 & \pm 11 & 0.303 & \pm 0.005 & 0.30 & \pm 0.06 \\
\hline pNA (ref) & \multirow{2}{*}{ 苂 } & 11.2 & \pm 1.6 & 4.6 & \pm 1.2 & 2.1 & \pm 2.6 & 8.2 & \pm 1.2 & 0.22 & \pm 0.030 & 0.30 & \pm 0.06 \\
\hline $\mathrm{CV}$ & & 305 & \pm 58 & 341 & \pm 72 & 276 & \pm 52 & 398 & \pm 81 & 0.65 & \pm 0.02 & 0.67 & \pm 0.08 \\
\hline pNA (ref) & \multirow{6}{*}{ 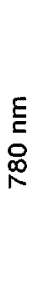 } & 56.4 & \pm 5.9 & 2 & \pm 18 & 23.4 & \pm 3.8 & 38.3 & \pm 4.1 & & & 0.24 & \pm 0.05 \\
\hline CV & & 552 & \pm 42 & 353 & \pm 34 & 309 & \pm 36 & 287 & \pm 31 & 0.74 & \pm 0.02 & 0.72 & \pm 0.04 \\
\hline MG & & 122 & \pm 10 & 162 & \pm 18 & 120 & \pm 13 & 131 & \pm 14 & 1.27 & \pm 0.03 & 1.45 & \pm 0.11 \\
\hline BG & & 142 & \pm 11 & 186 & \pm 16 & 136 & \pm 12 & 153 & \pm 13 & 1.40 & \pm 0.05 & 1.43 & \pm 0.03 \\
\hline RT9090 & & 278 & \pm 21 & 112 & \pm 27 & 135 & \pm 19 & 198 & \pm 16 & 0.39 & \pm 0.01 & 0.40 & \pm 0.04 \\
\hline $1955-49$ & & 289 & \pm 22 & 119 & \pm 25 & 167 & \pm 16 & 200 & \pm 16 & 0.46 & \pm 0.01 & 0.46 & \pm 0.04 \\
\hline
\end{tabular}

tensor $\beta$ 's having the same symmetry and not the others. Consequently, it is possible to define optimization conditions for each part of $\chi$ separately without knowing the precise form of the orientational distribution function. The knowledge of the symmetry group of the chromophore and the medium can predict that some of the four parts in (8) must vanish identically. For example, if the symmetry of the aligned medium is such that $\chi$ automatically satisfies Kleinman (full permutation) symmetry, the Kleinman nonsymmetric parts $2 m$ and $1 m$ in (8) will have to vanish due to alignment even if the corresponding molecular characteristics are not zero. Since the goal of this study is to achieve optimization of the NLO response through nonpolar alignment, the main focus of the further discussion will be directed at the nonvector irreducible components of $\chi$.

We consider the two most important cases of chiral axial systems - uniaxially aligned media with $D_{\infty}$ symmetry and biaxial media with $D_{2}$ symmetry. These two types of alignment are chosen because they represent a wide range of physical systems that can be easily fabricated. The first type of alignment can be realized in stretched polymers or in uniaxial liquid crystal phases (e.g., nematic or smectic $A$ ). The second alignment scheme occurs in more ordered systems like biaxial liquid crystal phases (e.g., smectic $C$ ) or liquid-crystalline polymers, where two distinct directions are defined by the polymer's backbone and liquid-crystalline side-chain moieties. Clearly, the chirality of both symmetry groups requires that measures be taken to assure the absence of the mirror plane symmetry in the bulk. Chirality of the bulk does not require chiral chromophores, and can be achieved by aligning achiral chromophores in a chiral fashion, as will be illustrated below.
In general, there can be a macroscopic hyperpolarizability coming from the $2 m$ terms only if a) the chromophores have a symmetry such that there is at least one traceless symmetric second-rank pseudotensor that does not change under all the allowed symmetry elements and b) if the same is true of the macroscopic medium. In more technical group-theoretical terms, the representation of the traceless symmetric second-rank pseudotensor must contain the trivial representation at least once in the symmetry groups of both the chromophore and the medium.

\section{A. Uniaxial Alignment of $\Lambda$-Chromophores- $C_{2 v}$ Chromophore and $D_{\infty}$ Symmetry of the Medium}

In the uniaxial alignment scheme $D_{\infty}$, the nonlinear susceptibility is fully defined by one value. In Cartesian coordinates (with $z$ being the unique axis), this is $\chi\left(D_{\infty}\right)=\chi_{x y z}$ $=\chi_{x z y}=-\chi_{y z x}=-\chi_{y x z}$, while all the other components vanish. The analysis of the irreducible content of the susceptibility tensor $\chi$ shows that there is only the second-rank component that is nonzero in the decomposition of (8)

$$
\chi^{(2 m)}=\chi_{x y z}\left(\begin{array}{rrr}
-1 & 0 & 0 \\
0 & -1 & 0 \\
0 & 0 & 2
\end{array}\right) .
$$

The optimization of nonlinear response of a $D_{\infty}$ medium can only be achieved by maximizing the second-rank hyperpolarizability of the chromophore and by choosing the type of alignment that is compatible with the symmetry of this component. 


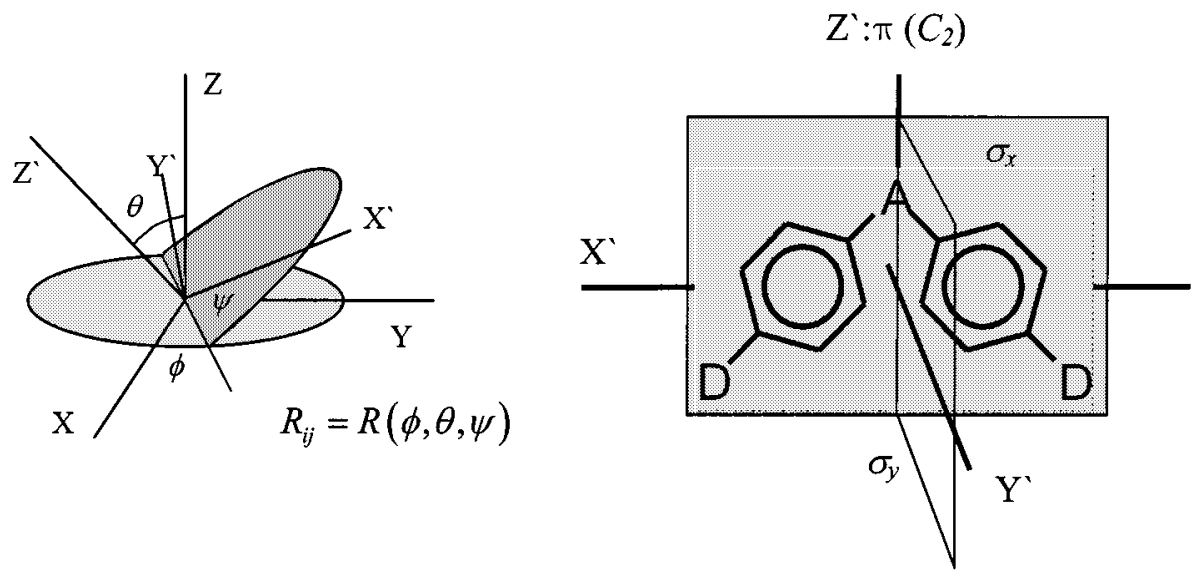

Fig. 3. Euler angle rotation and molecular coordinates for a $\Lambda$-shaped molecule.

First, consider the more general case of an arbitrary chromophore's being aligned into $D_{\infty}$ bulk. The only requirement imposed on the molecule is the existence of nonzero $\beta^{(2 m)}$. Using the permutation properties of $\chi\left(D_{\infty}\right)$, one can write it as

$$
\begin{aligned}
\chi_{D_{\infty}}^{(2 m)} & =\frac{1}{6}\left(2 \chi_{z z}-\chi_{x x}-\chi_{y y}\right) \\
& =\frac{1}{6} N\left\langle 2 R_{z i} R_{z j}-R_{x i} R_{x j}-R_{y i} R_{y j}\right\rangle \beta_{i j}^{(2 m)} .
\end{aligned}
$$

Here, the hyperpolarizability given in molecular frame is transformed into laboratory coordinates with orthogonal rotation matrix $\overleftrightarrow{R}_{i j}=\hat{i} \cdot \hat{j}^{\prime}\left(\hat{i}\right.$ and $\hat{j}^{\prime}$ stand for basis vectors of laboratory and molecular frame, respectively). In writing (10), we could have chosen to calculate any nonzero combination of the tensor hyperpolarizability. The particular form that we chose above, which explicitly projects the specific traceless symmetric tensor that is nonzero in this macroscopic medium, is convenient, as it makes the nature of the average more evident in subsequent steps.

More specific knowledge of the chromophore's hyperpolarizability or the constraints imposed thereon by symmetry allows one to draw conclusions about alignment from (10). As mentioned above, $\Lambda$-shaped chromophores may exhibit large Kleinman-disallowed hyperpolarizabilities. Most $\Lambda$-shaped molecules can be approximated well as a planar (two-dimensional) $C_{2 v}$ object so that the only nonzero Cartesian components of $\beta$ are $\beta_{z z z}, \beta_{z x x}$, and $\beta_{x z x}=\beta_{x x z}$ (assuming $z$ is the two-fold axis and $z x$ is the plane of the molecule). The second-rank tensor is given by

$$
\beta_{C_{2 v}}^{(2 m)}=\left(\begin{array}{ccc}
0 & \Delta \beta / 2 & 0 \\
\Delta \beta / 2 & 0 & 0 \\
0 & 0 & 0
\end{array}\right), \quad \Delta \beta=\beta_{z x x}-\beta_{x x z} .
$$

Combining (10) and (11) results in the expression for the $\beta\left(C_{2 v}\right) \rightarrow \chi\left(D_{\infty}\right)$ alignment scheme. The rotation matrix $R$ can be defined in terms of Euler angles, thus making the order parameter a function of three orientation angles of the chromophore in the bulk. The convention for Euler angles and the orientation of the molecular symmetry axis of a $\Lambda$-like molecule used here is shown in Fig. 3. So the nonlinear susceptibility defined in (10) is now given by

$$
\begin{aligned}
\chi^{(2 m)}\left(C_{2 v} \rightarrow D_{\infty}\right) \\
=\frac{1}{6} N \Delta \beta\left\langle 2 R_{z x} R_{z y}-R_{x x} R_{x y}-R_{y x} R_{y y}\right\rangle \\
=\frac{1}{6} N \Delta \beta\left\langle 2\left(\hat{z} \cdot \hat{x}^{\prime}\right)\left(\hat{z} \cdot \hat{y}^{\prime}\right)-\left(\hat{x} \cdot \hat{x}^{\prime}\right)\left(\hat{x} \cdot \hat{y}^{\prime}\right)\right. \\
\left.\quad \quad-\left(\hat{y} \cdot \hat{x}^{\prime}\right)\left(\hat{y} \cdot \hat{y}^{\prime}\right)\right\rangle \\
=\frac{1}{4} N \Delta \beta\left\langle\sin ^{2}(\theta) \sin (2 \psi)\right\rangle .
\end{aligned}
$$

The conditions for maximizing the order parameter of such a system become clear from (12). The order parameter naturally does not depend on the azimuthal angle $\phi$, since the system is invariant under this rotation. The macroscopic response of the system is maximized by the combination of the other two angles $\theta=\pi / 2$ and $\psi= \pm \pi / 4$. In terms of molecules' orientation, the situation corresponds to the polar axes of the molecules' being perpendicular to the $C_{\infty}$-axis of the bulk and twisted about their $C_{2}$ axes by $45^{\circ}$. Note that the direction of the twist must be the same: the sign of $\psi$ has to be chosen either plus or minus. The optimized alignment schemes for $\Lambda$-shaped chromophores in a uniaxial medium are summarized in Fig. 4.

\section{B. Biaxial Alignment of $\Lambda$-Chromophores- $C_{2 v}$ Chromophore and $D_{2}$ Symmetry of the Medium}

Consider the second chiral alignment type of interest: $-D_{2}$ symmetry. The polar axis is also absent in this case, so that the vector components in (8) vanish identically as in the previous case. The second- and third-rank tensors, however, are allowed in $D_{2}$. The tensor is defined in terms of three values (nonzero Cartesian component $\chi_{x y z}=\chi_{x z y}, \chi_{y z x}=\chi_{y x z}$, and $\chi_{z x y}=\chi_{z y x}$ ). In terms of irreducible tensors, the fully symmetric octupolar part of the susceptibility tensor $\chi$ is defined by a single value, while the second-rank part carries two others

$$
\begin{aligned}
\chi^{s} & =\chi_{x y z}^{(3 s)}=\chi_{y z x}^{(3 s)}=\cdots=\frac{1}{3}\left(\chi_{x y z}+\chi_{y z x}+\chi_{z x y}\right) \\
\chi^{(2 m)} & =\left(\begin{array}{ccc}
\chi_{y z x}-\chi_{z x y} & 0 & 0 \\
0 & \chi_{z x y}-\chi_{x y z} & 0 \\
0 & 0 & \chi_{x y z}-\chi_{y z x}
\end{array}\right) .
\end{aligned}
$$


The second-rank tensor in (13) can be split into two parts, which we will call uniaxial and biaxial

$$
\begin{aligned}
\chi^{(2 m)} & =\chi^{u}\left(\begin{array}{rrr}
-1 & 0 & 0 \\
0 & -1 & 0 \\
0 & 0 & 2
\end{array}\right)+\chi^{b}\left(\begin{array}{rrr}
-1 & 0 & 0 \\
0 & 1 & 0 \\
0 & 0 & 0
\end{array}\right) \\
\chi^{u} & =\frac{\chi_{x y z}-\chi_{y z x}}{2} ; \quad \chi^{b}=\frac{2 \chi_{z x y}-\chi_{x y z}-\chi_{y z x}}{2} .
\end{aligned}
$$

The uniaxial term of (14) is identical in structure to $\chi_{D_{\infty}}^{(2 m)}$ [(9)] with the exception of a differently defined scalar order parameter [which, in fact, transforms into (9) upon transition of $D_{2}$ to $D_{\infty}$ ]. Therefore, the entire discussion of the optimization of the macroscopic response in $D_{\infty}$ media is fully applicable to the uniaxial part of $\chi^{(2 m)}$ so that the conditions for maximizing the order parameter $\chi^{u}$ are given by (12). The second term of (14) corresponds to biaxial alignment. The third independent value in $\chi\left(D_{2}\right)$ can be defined as follows:

$$
\chi^{b}=\frac{1}{2} N\left\langle R_{y i} R_{y j}-R_{x i} R_{x j}\right\rangle \beta_{i j}^{(2 m)} .
$$

In the special case of $C_{2 v}$ chromophore [(11)], the biaxial part of the second-rank susceptibility is given by

$$
\begin{aligned}
\chi^{b} & =\frac{1}{2} N \Delta \beta\left\langle R_{y x} R_{y y}-R_{x x} R_{x y}\right\rangle \\
& =\frac{1}{2} N \Delta \beta\left\langle\left(\hat{y} \cdot \hat{x}^{\prime}\right)\left(\hat{y} \cdot \hat{y}^{\prime}\right)-\left(\hat{x} \cdot \hat{x}^{\prime}\right)\left(\hat{x} \cdot \hat{y}^{\prime}\right)\right\rangle \\
& =\frac{1}{16} N \Delta \beta\langle 4 \cos (\theta) \cos (2 \psi) \sin (2 \phi)+(3+\cos (2 \theta)) \\
& \cdot \sin (2 \psi) \cos (2 \phi)\rangle .
\end{aligned}
$$

The orientational average in (16) can be maximized if the preferred polar angle of the chromophore $\theta$ is close to zero or $\pi$. Equation (16) reduces to

$$
\begin{aligned}
& \chi^{b}(\theta=0)=\frac{1}{4} N \Delta \beta\langle\sin [2(\psi+\phi)]\rangle \\
& \chi^{b}(\theta=\pi)=\frac{1}{4} N \Delta \beta\langle\sin [2(\psi-\phi)]\rangle .
\end{aligned}
$$

When the polar angle is zero, the azimuthal rotation and twist about the molecule's axis become equivalent, so that only the sum $\psi+\phi$ describes the orientation of the molecule. When $\theta=\pi$, the molecules are turned upside down between the first and the last rotations. The first and last rotations are also coaxial here, although positive values of $\psi$ correspond to negative values of $\phi$ and the twist angle is defined as the difference $\psi-\phi$. Therefore, the maximum of the biaxial component of the susceptibility can be achieved when the molecules are aligned along an axis pointing either up or down with the plane of the molecules twisted $45^{\circ}$ in opposite directions for upward and downward pointing species, so that their molecular planes ( $x z-$ in Fig. 3) are perpendicular.

\section{Propeller-Like Molecules ( $D_{n}$ Chromophores in $D_{m}$}

Media, $n, m>2$ )

Another example that we will discuss in relation to the axial alignment of Kleinman asymmetric chromophores is systems built with molecules of $D_{3}$ symmetry group that are aligned in $D_{\infty}$ fashion. Recently, various three-fold propeller-like NLO chromophores have been extensively studied in relation to their use in octupolar NLO media (e.g., [29] and [30]). However, if

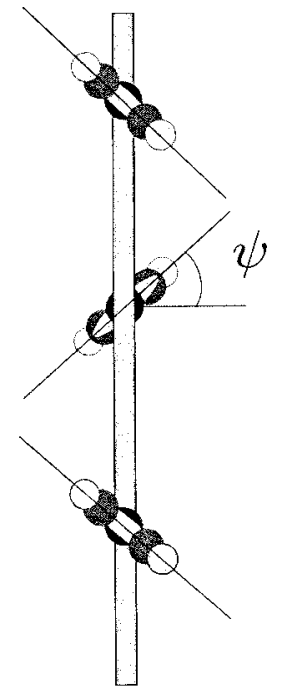

a)

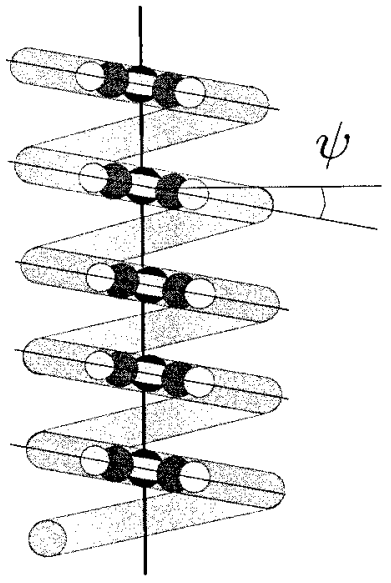

b)
Fig. 4. Example alignment of $\Lambda$-shaped molecules in a uniaxial macroscopic NLO system.

the chromophore is not perfectly flat, its symmetry is compatible with the second-rank irreducible component, which may be rather large, as is indicated by our results for Crystal Violet [15] (which, however, does not have its expected $D_{3}$ symmetry in the medium in which we have done measurements). Here we present an alignment scheme where the performance of the material is based on Kleinman-disallowed $\beta^{(2 m)}$ rather than octupolar part $\beta^{(3 s)}$.

The symmetry group $D_{3}$ is a uniaxial group with the second-rank hyperpolarizability tensor identical in structure to (9)

$$
\beta_{D_{3}}^{(2 m)}=\beta_{x y z}\left(\begin{array}{rrr}
-1 & 0 & 0 \\
0 & -1 & 0 \\
0 & 0 & 2
\end{array}\right) .
$$

The rotational averages are even more straightforward to interpret in this case

$$
\begin{aligned}
\chi & \left(D_{3} \rightarrow D_{\infty}\right) \\
\quad & =\chi_{x y z}=\frac{1}{6} N\left\langle 2 R_{z i} R_{z j}-R_{x i} R_{x j}-R_{y i} R_{y j}\right\rangle \beta_{i j}^{D_{3}} \\
& =\frac{1}{2} N \beta_{x y z}\left\langle 2 R_{z z} R_{z z}-R_{x z} R_{x z}-R_{y z} R_{y z}\right\rangle \\
& =\frac{1}{4} N \beta_{x y z}\langle 1+3 \cos (2 \theta)\rangle .
\end{aligned}
$$

The optimal response of such a system is achieved with all molecules lying in the plane perpendicular to the symmetry axis of the bulk $(\theta=0, \pi)$. The azimuthal alignment does not influence this part of the nonlinear susceptibility. The uniaxial alignment makes use of only the chiral $(2 \mathrm{~m})$ part of the hyperpolarizability of a $D_{n}(n>2)$ molecule $\left(\beta_{x y z}\right)$, while the utilization of the other, octupolar $(3 s)$, nonzero component $\left(\beta_{x x x}=-\beta_{x y y}=-\beta_{y x y}=-\beta_{y y x}\right)$ would require stricter, three-fold alignment that is generally harder to achieve in molecular media. A sketch of the required order is depicted in Fig. 5. 


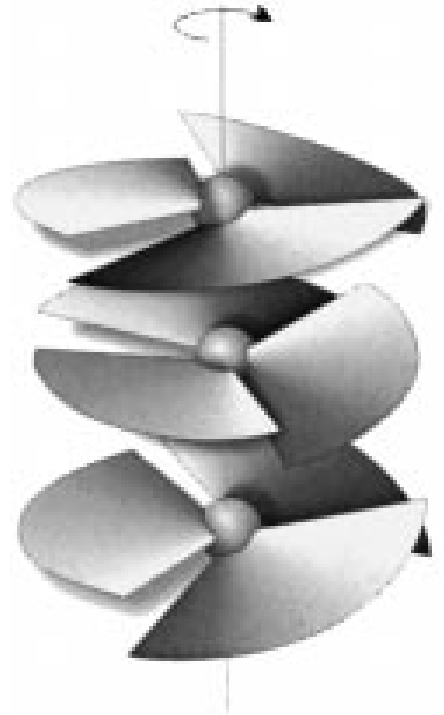

Fig. 5. Alignment of three-fold propeller-like chromophores in a bulk of $D_{\infty}$ symmetry.

Biaxial alignment of propeller molecules is also possible. However, we know of no polymeric materials that plausibly have this symmetry and in consequence do not discuss it.

\section{DISCUSSION}

The previous section discussed how chiral macroscopic NLO systems could be created via chiral alignment of chromophores that belong to a different symmetry group but contain Kleinmandisallowed (second-rank) contribution in the molecular hyperpolarizability. A set of molecular orientation angles that optimizes the macroscopic response of the material was deduced on the basis of general symmetry considerations for each case of interest. The rotational freedom of the chromophore has to be limited, although it may freely rotate around a particular axis in the uniaxial case. As discussed above, the alignments described can be achieved relatively easily in polymer systems with NLO chromophores incorporated in the backbone or attached as a side chain.

A number of chiral polymeric systems for nonlinear optics have been studied recently. Van Elshocht $e t$ al. studied NLO response of Langmuir-Blodgett films made of binaphthyl-based chiral helical polymers [9]. However, these systems do not seem to be optimized in the axial sense discussed here. The authors report the symmetry of the films to be $C_{\infty}$, which is supported by the observation that $\chi_{z z z}$ is the largest component of the hyperpolarizability. Indeed, in the materials presented, the $\Lambda$-shaped monomers are attached to each other with their "feet" creating the polymer main chain, so that in the aligned state, they would rather have the planes of the molecules aligned parallel to the polymer's axis than at the $45^{\circ}$ angle - the condition that would favor large hypersusceptibility resulting from a traceless symmetric tensor hyperpolarizability.

Another work by Kauranen et al. presents a study of the NLO properties of helicenebisquinone-based Langmuir-Blodgett films [31]. The enantiomerically pure chiral material aggregates into a film of $C_{2}$ symmetry. However, the measurements of the nonlinear susceptibility $\chi$ showed that by far the largest Cartesian components are $\chi_{x y z}$ and $\chi_{y x z}$, i.e., the characteristic components of chiral groups $D_{\infty}$ and $D_{2}$ discussed here. So, one can imagine (at least in rough approximation) that the molecules pack in helical columns creating a structure with $D_{\infty}$ symmetry (or some similar uniaxial dihedral group). In this case, the chirality of the bulk is forced directly by the strong chirality of the helicenebisquinone. The molecule is essentially three-dimensional and, although clearly it is not the $C_{2 v}$ case discussed in most detail above, the symmetry of the chromophore is the simpler group $C_{2}$ that has similar properties. The chromophore can also be thought of as a $\Lambda$-shaped chromophore. As they are stacked in columns, the molecules automatically satisfy the condition for the polar angle (pointing perpendicular to the column axis). At the same time, the "plane" of the molecule (i.e., the plane that cuts through the molecule's midpoint — the "vertex"—and two end points - the "feet" of $\Lambda$ ) is naturally twisted by some (not 0 or $90^{\circ}$ ) angle away from the normal to the bulk principal axis due to the pitch of the chiral column. Moreover, the axes $x$ and $y$ discussed above in conjunction with $C_{2 v}$ molecules will be rotated by some hard-to-discern angle, which is likely to vary with frequency from (say) the plane of the central aromatic ring. Thus, given the formalism presented above, the large chiral nonlinearity reported in [31] can be accounted for by satisfactorily (if not optimally) aligned $\Lambda$-shaped molecules of helicenebisquinone.

The desired alignment of $\Lambda$-shaped chromophores can be also achieved by other means. For a $D_{\infty}$ system, a material consisting of long, linear polymers that have $\Lambda$-shaped chromophores attached to the backbone with their vertices can be stretched in one direction, so that the molecular dipoles are, generally, perpendicular to the main chain. The linking group must contain a chiral center that favors the rotation of the plane of the chromophore by $\sim 45^{\circ}$ from the direction of the main chain of the polymer, with the most important condition being that a preponderance of the chromophores are twisted in the same direction. An alternative scheme is somewhat similar to the system in [31]: the chromophores are attached (again at their vertices) to a helical polymer backbone heading toward the helix axis. The rotation about the molecular axes must still be constrained, although it can be parallel to the local direction of the polymer chain and only tilt with respect to the symmetry axis is created (and even controlled) by pitch of the helix. The two cases are schematically shown in Fig. 4.

The case of $D_{2}$ alignment is slightly more complicated. The structure is similar to the first example of $D_{\infty}$ alignment from the previous paragraph. The chromophores are incorporated into a linear polymer as side-chain groups with a fixed twist angle. In addition, liquid crystalline moieties are attached to the "feet" of the $\Lambda$-shaped chromophore. When conditions are satisfactory for existence of the nematic phase, the direction of the stretch (along the polymer chain) and director of the nematic phase (perpendicular to the main axis) define two distinct nonpolar axes in the system, forming $D_{2}$ symmetry. When the nematic moieties align parallel to each other, the chromophores will, on average, have to point mostly "up" and "down," favoring only $\theta=0, \pi$. Such alignment can also, in principle, be achieved 


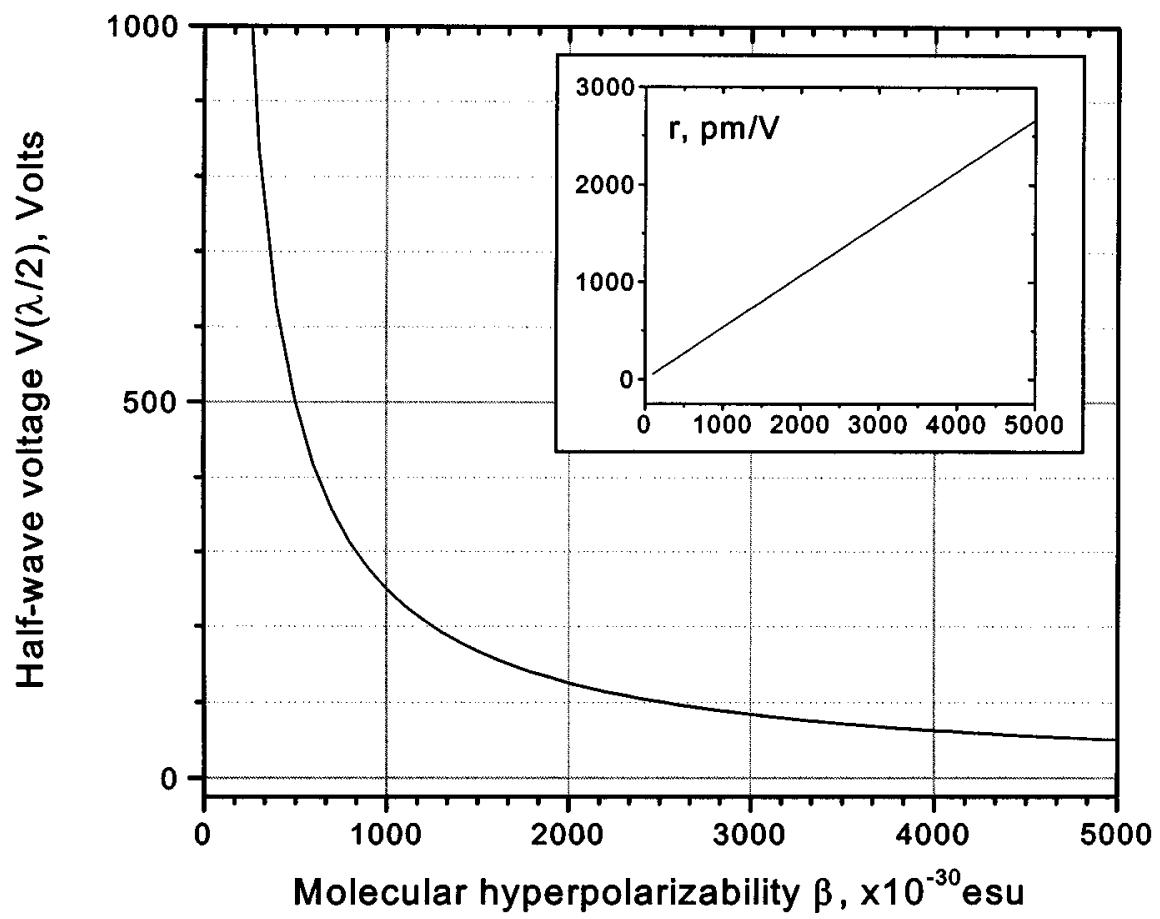

Fig. 6. Half-wave voltage of a longitudinal EO modulator (electrooptic coefficient inset).

by stretching of an appropriately cross-linked polymer gel in a biaxial fashion.

Finally, the optimized alignment scheme for $D_{3}$ chromophores as depicted in Fig. 5 can be physically realized in discotic nematic or columnar liquid crystals or LC-polymers. Here the chirality of such systems should originate from the chirality of the chromophores. Hence, an enantiomerically pure chromophore should be used for the best performance, since the left- and right-handed species, if present simultaneously, will cancel each other's hyperpolarizability, so that the hyperpolarizability is proportional to the enantiomeric excess. Molecules with a low threshold for the transition between leftand right-handed states (like Crystal Violet) can be stabilized if placed into a matrix (or a host liquid crystal) with a well-defined handedness, or, if appropriate, chiral optically unresponsive moieties are attached thereto.

\section{Device Prospects}

Speculation on the prospects for the application of axially ordered chiral nonlinear optical media begins with the advantages one gains by using nonpolar media. The alignment schemes discussed above have very different and apparently much less stringent constraints on chromophore density and alignment than the usual polar alignment. For example, one might consider pure discotic columnar liquid crystal materials composed of $D_{3}$ symmetry chromophores, such as shown in Fig. 5. In a discotic liquid crystal, as much as $40 \%$ of the molecular mass can be directly involved in the optically responsive part of the chromophore. This is to be compared with $<20 \%$ in most poled polymers. The order parameters in nematic liquid crystals vary, but values of $0.8-0.9$ are easily achievable, with higher values routinely achieved in polymeric systems. Again in a poled polymer system, $20 \%$ would be a large-order parameter. Thus, a highly ordered and dense collection of nonlinear optical chromophores should be reasonably easy to achieve.

We now consider a linear electrooptic device, such as a modulator. Given the usual values of the linear electrooptic coefficient $\left(r_{\text {eff }}<100 \times 10^{-12} \mathrm{~m} / \mathrm{V}\right)$, either the half-wave modulation voltage is large $(<1000 \mathrm{~V})$ or the interaction length is made long in a transverse modulator configuration by using a waveguide geometry in order to use a low half-wave voltage. For example, sub-1-V modulation voltages have been demonstrated in guided-wave devices using polar electrooptic polymers [32]-[34]. We consider here a longitudinal modulator, i.e., where the propagation direction of the light is generally along the direction of the modulating electric field. In this configuration, the half-wave voltage is independent of the propagation length. One can then consider transmission of light through a thin film, forming an image plane or spatial light modulator (SLM). For usual electronic linear electrooptic coefficients, however, such a configuration requires a very high half-wave voltage, which generally requires the much larger responses found in liquid crystal SLMs. These liquid crystal devices have large responses based on the coupling of the modulating field to the elastic properties of the liquid crystal. Although these effects are large, they are rather slow, generally on the millisecond time scale, or on the microsecond time scale in ferroelectric liquid crystals.

We now estimate the half-wave voltage in a longitudinal modulator configuration for the responses discussed in this paper. We can estimate the linear electrooptic coefficient $r_{\text {eff }}$ from the molecular hyperpolarizability, density, order parameter, and local fields. In this case, the effective electrooptic coefficient is given by

$$
r_{\mathrm{eff}}=\frac{2 d}{n^{4}}=\frac{2}{n^{4}} N f^{0}\left(f^{\omega}\right)^{2} g \beta
$$




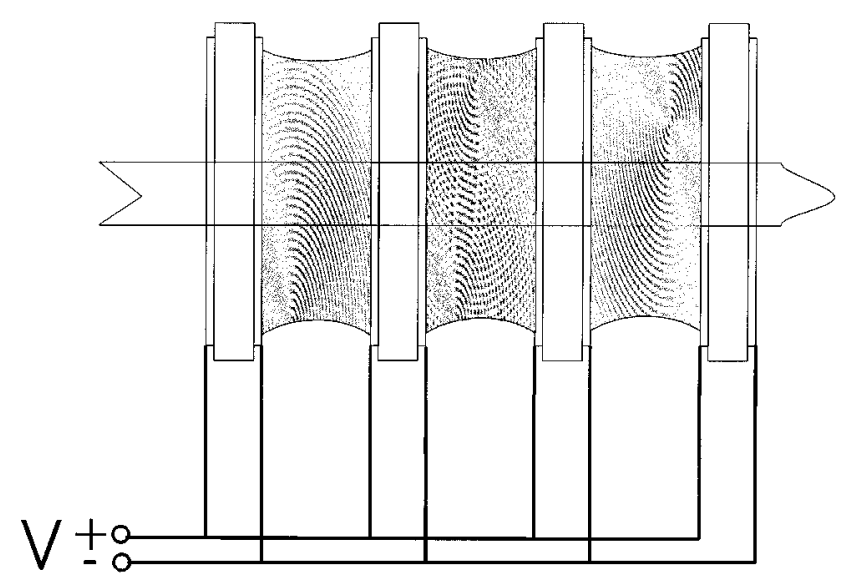

Fig. 7. Schematic of a multilayer EO spatial light modulator with reduced half-wave voltage.

where we have ignored dispersion in the refractive index and where $N$ is the number density of molecules, $f \mathrm{~s}$ are local field factors at the appropriate frequencies, $g$ is the order parameter, and $\beta$ is the hyperpolarizability figure of merit as measured by KD-HRS. The local field factors are given by

$$
f^{0}=\frac{\varepsilon\left(n^{2}+2\right)}{n^{2}+2 \varepsilon} ; \quad f^{\omega}=\frac{n^{2}+2}{3} .
$$

The half-wave voltage is then given by

$$
V_{1 / 2}=\frac{\lambda}{n^{3} r_{\mathrm{eff}}}
$$

where $\lambda$ is the wavelength. Fig. 6 depicts the dependence of the half-wave voltage and the linear electrooptic coefficient $r_{\text {eff }}$ for such a device. We have assumed material values appropriate for a neat liquid crystal, i.e., $N=1.2 \cdot 10^{21} \mathrm{~cm}^{-3}, n=1.65$, $g=0.9, \lambda=600 \mathrm{~nm}$, and $\varepsilon=4$. We remark that the results depicted in Table I indicate values of hyperpolarizability in the hundreds (in units of $10^{-30} \mathrm{esu}$ ). However, these results are initial experiments performed on available or easy to make molecules to demonstrate the symmetry concepts presented here: little work has gone into synthetic, experimental, and quantum chemical studies aimed at optimizing the transition moments responsible for the relevant molecular response. Such work has greatly increased the vector hyperpolarizabilities. It is reasonable to suppose that such studies will result in optimized structures with responses comparable to the best figures of merit for the vector hyperpolarizability. Figures of merit exceeding 1000 have been observed [34]-[36] for the vector hyperpolarizability. Indeed, our results as indicated in Table I indicate that $2 \mathrm{~mm}$ and $3 \mathrm{ss}$ figures of merit may be routinely comparable to the $1 s s$ component.

As we have mentioned in the longitudinal modulator geometry, the half-wave voltage is independent of thickness so that very thin devices might be constructed. In this case, absorption losses become much less problematic, and one may be able to exploit resonant nonlinear optical response. In this case, figures of merit greater than $2000 \times 10^{-30}$ esu may be possible, leading to switching voltages under $100 \mathrm{~V}$. Additionally, multilayer devices as depicted in Fig. 7 may be constructed to have switching voltages below $10 \mathrm{~V}$. Since the effects described here are purely electronic, we expect ultrafast response times for electrooptic switching, so that the device response time is likely to be limited by the electronics. As uncharged polymers are known to have relatively low dielectric constants, the electronics can, likely, be quite fast. Thus, we speculate that this approach to electrooptic materials may allow production of SLMs with gigahertz bandwidths, with the bandwidth limited by the capactance, which can be reduced in a pixel structure. Such devices would have a number of interesting applications in the electronics industry.

\section{CONCLUSION}

In this paper, we have discussed prospects for creating a new class of second-order nonlinear optical media based on axially ordered nonpolar chiral media. We have discussed molecular and supramolecular criteria for optimizing the nonlinear optical response in such media. On the molecular level, we have discussed symmetry considerations and described several possible interesting symmetry groups. We have demonstrated these concepts in some model $\Lambda$-like chromophores. Our results have indicated that technologically interesting molecular figures of merit are possible. Indeed, it seems that figures of merit comparable to the current polar materials are routine. We have also shown how bulk nonlinear susceptibility can be created in chiral nonpolar media. The theory that elucidates the criteria for optimization of the nonlinear response of such chiral media has been summarized. Based on the conclusions drawn from the theory, several examples of known materials as well as possible physical configurations involving $\Lambda$-shaped and propeller-like chromophores were discussed. We have also speculated on how such materials may be applied to longitudinal electrooptic spatial light modulators. It seems that gigahertz bandwidth SLMs with switching voltages under $10 \mathrm{~V}$ may be possible. Our results suggest that further work may lead to an interesting nonlinear optical material technology.

\section{REFERENCES}

[1] G. A. Lindsay and K. D. Singer, Eds., Polymers for Second-Order Nonlinear Optics. Washington, DC: ACS Symp. Ser. 601, 1995.

[2] J. Zyss, Ed., Molecular Nonlinear Optics. Boston, MA: Academic, 1994.

[3] J. A. Giordmaine, "Nonlinear optical properties of liquids," Phys. Rev., vol. 138, pp. 1599-1606, Mar. 1965.

[4] R. Andreu, I. Malfant, P. G. Lacroix, H. Gornitzka, and K. Nakatani, "Chiral stilbazolium chromophores: An approach toward multiproperty materials combining conductivity and second-order optical nonlinearities," Chem. Mater., vol. 11, pp. 840-848, Mar. 1999.

[5] N. Koroteev, V. A. Makarov, and S. N. Volkov, "Second-harmonic generation in the bulk of a chiral liquid by a focused laser beam," Opt. Commun., vol. 157, pp. 111-114, Dec. 1998.

[6] S. Wozniak and G. Wagniere, "Optically induced second harmonic generation in chiral liquids," Opt. Commun., vol. 151, pp. 81-85, May 1998.

[7] F. Steybe, F. Effenberger, U. Gubler, C. Bosshard, and P. Günter, "Highly polarizable chromophores for nonlinear optics: Syntheses, structures and properties of donor-acceptor substituted thiophenes and oligothiophenes," Tetrahedron, vol. 54, pp. 8469-8480, Jul. 1998.

[8] S. Van Elshocht, T. Verbiest, M. Kauranen, A. Persoons, B. M. W. Langeveld, Voss, and E. W. Meijer, "Direct evidence of the failure of electric-dipole approximation in second-harmonic generation from a chiral polymer film," J. Chem. Phys., vol. 107, pp. 8201-8203, Nov. 1997.

[9] S. Van Elshocht, T. Verbiest, M. Kauranen, L. Ma, H. Cheng, K. Y. Musick, L. Pu, and A. Persoons, "Chiral 1,1'-binaphthyl-based helical polymers as nonlinear optical materials," Chem. Phys. Lett., vol. 309, pp. 315-320, Aug. 1999. 
[10] D. M. Walba, D. J. Dyer, X. H. Chen, U. Muller, P. Cobben, R. F. Shao, and N. A. Clark, "Design and synthesis of ferroelectric liquid crystals 0.25. An approach to new materials for ultra-fast electronic electro-optic modulators," Mol. Cryst. Liq. Cryst. Sci. Tech., vol. 288, pp. 83-91, 1996.

[11] Y. Luo, M. Lindgren, and H. Agren, "Nonlinear optical properties of multi-dimensional charge transfer functional groups in molecules designed for liquid crystals," Opt. Mater, vol. 9, pp. 216-219, Jan. 1998.

[12] V. Ostroverkhov, O. Ostroverkhova, R. G. Petschek, K. D. Singer, L. Sukhomlinova, R. J. Twieg, S.-X. Wang, and L. C. Chien, "Optimization of the molecular hyperpolarizability for second harmonic generation in chiral media," Chem. Phys., vol. 257, pp. 263-274, July 2000.

[13] V. R. Thalladi, S. Brasselet, H.-C. Weiss, D. Blaster, A. K. Katz, H. L. Carrell, R. Boese, J. Zyss, A. Nangia, and G. R. Desiraju, "Crystal engineering of some 2,4,6-triaryloxy-1,3,5-triazines: Octupolar nonlinear materials," J. Amer. Chem. Soc., vol. 120, pp. 2563-2577, 1998.

[14] S. F. Hubbard, R. G. Petschek, K. D. Singer, N. D’Sidocky, C. Hudson, L. C. Chien, C. C. Henderson, and P. A. Cahill, "Measurements of Kleinman-disallowed hyperpolarizability in conjugated chiral molecules," J. Opt. Soc. Amer. B, vol. 15, pp. 289-301, Jan. 1998

[15] V. Ostroverkhov, R. G. Petschek, K. D. Singer, L. Sukhomlinova, R. J. Twieg, S.-X. Wang, and L. C. Chien, "Measurements of the hyperpolarizability tensor by means of hyper-Rayleigh scattering," J. Opt. Soc. Amer. B, vol. 17, pp. 1531-1542, Sept. 2000.

[16] J. Jerphagnon, D. Chemla, and R. Bonneville, "The description of the physical properties of condensed matter using irreducible tensors," $A d v$. Phys., vol. 27, pp. 609-650, 1978.

[17] D. A. Kleinman, "Nonlinear dielectric polarization in optical media," Phys. Rev., vol. 126, pp. 1977-1979, 1962.

[18] R. W. Boyd, Nonlinear Optics. New York: Academic, 1992.

[19] J. L. Oudar and J. Zyss, "Structural dependence of nonlinear-optical properties of methyl-(2,4-dinitrophenyl)-aminopropanoate crystals," Phys. Rev. A, vol. 26, pp. 2016-2027, 1982.

[20] R. Wortmann, P. Krämer, C. Glania, S. Lebus, and N. Detzer, "Deviations from Kleinman symmetry of the second-order polarizability tensor in molecules with low-lying perpendicular electronic bands," Chem. Phys., vol. 173, pp. 99-108, 1992.

[21] C. R. Moylan, S. Ermer, S. M. Lovejoy, I.-H. McComb, D. S. Leung, R. Wortman, P. Krdmer, and R. J. Twieg, "(Dicyanomethylene)pyrane derivatives with $C_{2 v}$ symmetry: An unusual class of nonlinear chromophores," J. Amer. Chem. Soc., vol. 118, pp. 12950-12 955, 1996.

[22] V. Ostroverkhov, R. G. Petschek, K. D. Singer, and R. J. Twieg, "Lambda-like chromophores for chiral nonlinear optical materials," Chem. Phys. Lett., vol. 340, pp. 109-115, 2001.

[23] H. B. Lueck, J. L. McHale, and W. D. Edwards, "Symmetry-breaking solvent effects on the electronic structure and spectra of a series of triphenylmethane dyes," J. Amer. Chem. Soc., vol. 114, pp. 2342-2348, 1992.

[24] Y. Maruyama, M. Ishikawa, and H. Satozono, "Femtosecond isomerization of crystal violet in alcohols," J. Amer. Chem. Soc., vol. 118, pp. 6257-6263, 1996.

[25] P. Kaatz and D. P. Shelton, "Polarized hyper-Rayleigh light scattering measurements of nonlinear optical chromophores," J. Chem. Phys., vol. 105, pp. 3918-3929, 1996.

[26] K. D. Singer, S. F. Hubbard, A. Schober, L. M. Hayden, and K. Johnson, "Second harmonic generation," in Characterization Techniques and Tabulations for Organic Nonlinear Optical Materials, M. G. Kuzyk and C. W. Dirk, Eds. Berlin, Germany: Marcel Dekker, 1998, pp. $311-513$.

[27] J. L. Oudar and D. S. Chemla, "Hyperpolarizabilities of the nitroanilines and their relations to the excited state dipole moment," J. Chem. Phys., vol. 66, p. 2664, 1977.

[28] V. Ostroverkhov, K. D. Singer, and R. G. Petschek, "Second-order nonlinear optical processes in nonpolar chiral materials: Relationship between molecular and macroscopic properties," J. Opt. Soc. Amer. B, vol. 18 , pp. $1858-1865,2001$.

[29] M. Joffre, D. Yaron, R. J. Silbey, and J. Zyss, "Second order nonlinearity in octupolar aromatic systems," J. Chem. Phys., vol. 97, pp. 5607-5615, 1992.

[30] T. Verbiest, K. Clays, C. Samyn, J. Wolff, D. Reinhoudt, and A. Persoons, "Investigation of the hyperpolarizability in organic molecules from dipolar to octopolar systems," J. Amer. Chem. Soc., vol. 116, pp. 9320-9323, 1994.

[31] M. Kauranen, S. Van Elshocht, T. Verbiest, and A. Persoons, "Tensor analysis of the second-order nonlinear optical susceptibility of chiral anisotropic thin films," J. Chem. Phys., vol. 112, pp. 1497-1502, 2000.

[32] Y. Q. Shi, C. Zhang, H. Zhang, J. H. Bechtel, L. R. Dalton, B. H. Robinson, and W. H. Steier, "Low (sub-1-volt) halfwave voltage polymeric electro-optic modulators achieved by controlling chromophore shape," Science, vol. 288, pp. 119-122, Apr. 2000.
[33] Y. Q. Shi, W. P. Lin, D. J. Olson, J. H. Bechtel, H. Zhang, W. H. Steier, C. Zhang, and L. R. Dalton, "Electro-optic polymer modulators with 0.8 V half-wave voltage," Appl. Phys. Lett., vol. 77, pp. 1-3, Jul. 2000.

[34] L. R. Dalton, W. H. Steiner, B. H. Robinson, C. Zhang, A. Ren, S. Garner, A. Chen, T. Londergan, L. Irwin, B. Carlson, L. Fifield, G. Phelan, C. Kincaid, J. Amend, and A. Jen, "From molecules to opto-chips: Organic electro-optic materials," J. Mater. Chem., vol. 9, pp. 1905-1920, 1999.

[35] M. Blanchard-Desce, V. Alain, P. V. Bedworth, S. R. Marder, A. Fort, C. Runser, M. Barzoukas, S. Lebus, and R. Wortmann, "Large quadratic hyperpolarizabilities with donor-acceptor polyenes exhibiting optimum bond length alternation: Correlation between structure and hyperpolarizability," Chem. Eur. J., vol. 3, pp. 1091-1104, July 1997.

[36] C. H. Wang, J. N. Woodford, and A. K. Y. Jen, "Measurements of the first hyperpolarizabilities of thiophene-based charge-transfer chromophores with hyper-Rayleigh scattering at 1064 and $1907 \mathrm{~nm}$," Chem. Phys., vol. 262, pp. 475-487, Dec. 2000.

Victor Ostroverkhov received the Specialist degree in physics and optical engineering from National Taras Shevchenko University of Kyiv (Kiev), Ukraine, in 1996, and the Ph.D. degree in physics from Case Western Reserve University, Cleveland, $\mathrm{OH}$, in 2001.

His area of research is second-order nonlinear optics of organic materials, concentrated on aspects of chiral chromophores and nonpolar alignments.

Oksana Ostroverkhova received the Specialist degree in physics and optical engineering from National Taras Shevchenko University of Kyiv (Kiev), Ukraine, in 1996, and the Ph.D. in physics from Case Western Reserve University, Cleveland, OH, in 2001.

Currently, she is a Postdoctoral Researcher at the Department of Chemistry, Stanford University, Stanford, CA. Her research is on nonlinear optics of organic materials, including photorefractive effect in polymeric composites, photoelectric and charge transport properties, and surface nonlinear optics.

Rolfe G. Petschek received the B.S. degree in physics and in mathematics from the Massachusetts Institute of Technology, Cambridge, in 1975 and the Ph.D. degree in physics from Harvard University, Cambridge, MA, in 1981.

$\mathrm{He}$ is a Professor of Physics at Case Western Reserve University, Cleveland, $\mathrm{OH}$. He had postdoctoral appointments in chemistry at the University of California at Santa Barbara and San Diego before coming to Case Western Reserve in 1983. He has served as a Consultant for several companies.

Prof. Petschek is a member of the American Physical Society, the American Chemical Society, the International Liquid Crystal Society, and the International Society for Optical Engineering.

Kenneth D. Singer received the B. S. and Ph.D. degrees in physics from Ohio State University, Columbus, in 1975 and 1981, respectively.

$\mathrm{He}$ is a Professor of Physics and Macromolecular Science and Engineering at Case Western Reserve University (CWRU), Cleveland, OH. He was a Member of Technical Staff at Bell Laboratories from 1982 to 1989 and a Distinguished Member of Technical Staff from 1989 to 1990 . From 1990 to 1993, he was the Warren E. Rupp Associate Professor of Physics. He is currently Associate Chair of the Physics Department at CWRU and Director of the Engineering Physics program. His research interests are in organic optoelectronic materials, including nonlinear optical phenomena and electronic properties and transport in liquid crystals. He has received two patents on polymeric electrooptic materials and devices.

Prof. Singer is a Fellow of the Optical Society of America and a member of the American Physical Society and the International Liquid Crystal Society.

Ludmila Sukhomlinova received the B. S. degree in chemistry and the Ph.D. degree in organic chemistry from Moscow State University, Russia, in 1989 and 1993, respectively.

She is a Research Chemist at AlphaMicron Inc., Kent, OH. From 1994 to 1997, she held a permanent academic position at Moscow State University. In 1996-1997, she received a NATO Fellowship to conduct research at the Institute of Technology for Chemistry and Biology, New University of Lisbon, Portugal. From 1998 to 2000, she was a Postdoctoral Research Associate in the Chemistry Department at Kent State University. Her research interests include design and synthesis of organic optoelectronic materials.

R. J. Twieg, photograph and biography not available at the time of publication. 